\title{
A Hylleraas functional based perturbative technique to relax the Extremely Localized Molecular Orbital wavefunction
}

Alessandro Genoni ${ }^{(a, b) *}$, Kenneth M. Merz Jr. ${ }^{(a)}$, Maurizio Sironi ${ }^{(b)}$

(a) Quantum Theory Project, University of Florida, P.O. Box 118435, Gainesville, Florida, 32611, USA.

(b) Dipartimento di Chimica Fisica ed Elettrochimica, Università degli Studi di Milano, Via Golgi 19, 20133 Milano, Italy.

\begin{abstract}
A way to reduce the computational cost associated with the study of large molecules exploits the transfer of Extremely Localized Molecular Orbitals (ELMOs). Unfortunately, due to the reduction in the number of variational parameters, the ELMO description is not as accurate as the Hartree-Fock one, although it is qualitatively correct in most of the cases. Therefore, in order to overcome this significant drawback, we propose a perturbative ELMO approach exploiting the Hylleraas functional. Preliminary tests have been performed and the results are promising for future applications to large systems.
\end{abstract}

\section{Introduction}

One of the main contemporary challenges that theoretical chemists have to deal with involves the development of new techniques aimed at studying very large molecular systems and assemblies. This is why many groups have recently worked along these lines providing a plethora of promising methods, such as linear scaling techniques [1], which have had a prominent role in this context. Among them we highlight the "Divide and Conquer" strategy

\footnotetext{
*E-mail addresses: genoni@gtp.ufl.edu.
} 
[2-5], the Molecular Tailoring Approach [6-8] and the Adjustable Density Matrix Assembler (ADMA) method [9-11], techniques based on the idea that chemistry relies upon local concepts and that molecules can be "built up" from a suitable collection of fragments. In fact, all the linear scaling strategies have a sound physical basis in the "nearsightedness" of electronic structure [12-14], a principle that properly describes the weak interactions between very distant regions in a large system.

Another way by which the nearsightedness concept can be exploited to reduce the computational cost consists in the transfer of Localized Molecular Orbitals (LMOs) obtained through a posteriori [15] or a priori [16-19] techniques. In the former case, the canonical Hartree-Fock orbitals, which are completely delocalized over the whole system of interest, are subjected to unitary transformations to provide a new set of Localized Molecular Orbitals characterized by "orthogonalization tails" that make the resulting orbitals not readily transferable from one molecule to another one. Alternatively, in the a priori techniques, the presence of tails is avoided by defining a localization pattern based on chemical intuition before the calculations. In this way orbitals strictly localized on few atoms, namely Extremely Localized Molecular Orbitals or ELMOs, are obtained.

The first generalization of the Self Consistent Field equations for ELMOs was proposed by Stoll [16], and, using it, we have developed a robust algorithm for the determination of Extremely Localized Molecular Orbitals [20], algorithm that has been recently interfaced with the GAMESS-UK package [21]. Moreover, through a series of preliminary studies we have shown the transferability of ELMOs [20, 22-25, 28], which has stimulated us to develop DENPOL $[26,28]$, a program that allows for the computation of $a b$ initio quality electron densities for polypeptides through a database of suitable model compounds for all the aminoacids and a database of Extremely Localized Molecular Orbitals determined for these model molecules. Nevertheless, due to the ELMOs non-orthogonality, the calculation of the 
corresponding density matrix is not trivial and, for large systems, becomes too computationally demanding. Therefore, in order to speed up these computations we have combined the extremely localized nature of the ELMOs with the "Divide and Conquer" philosophy obtaining an approximate density matrix expression that allows for significant computational savings without affecting the accuracy of the results [27, 28]. Finally, in order to complete the overview about the ELMOs transferability, it is worth to observe that the Extremely Localized Molecular Orbitals have turned out to be proper orbitals to describe the frontier region in the mixed Quantum Mechanics / Molecular Mechanics methods [20, 29, 30] within the Local Self Consistent Field (LSCF) strategy developed by Assfeld and Rivail [31, 32].

Unfortunately, as already observed by several authors [33-44], an important problem connected with the ELMO description is that the energy associated with the ELMO wavefunction is, not unexpectedly, higher than the corresponding Hartree-Fock one. This is obviously due to the reduction in the number of variational parameters and we have already shown that, using the ELMOs as guess orbitals, just one/two traditional SCF iterations can largely reduce this energy difference $[22,28]$. Furthermore, in order to improve the electronic structure description provided by the ELMOs, we have devised a new strategy consisting in a non-orthogonal Configuration Interaction that exploits the extremely localized nature of the occupied and virtual orbitals to judiciously reduce the CI expansions [24, 25, 28].

In this paper we will propose an alternative approach to relax the wavefunction constructed with Extremely Localized Molecular Orbitals. In particular, starting from the seminal ideas in the old PCILO methods [33-40] and in the perturbation strategies by Nagata et al. [45, 46] and by Khaliullin et al. $[47,48]$ recently developed in the framework of the SCF-MI method $[49,50]$, we have developed a Hylleraas functional [51, 52] based perturbative technique and we present preliminary tests which show promise for future application of the new strategy to 
large molecular systems. It is extremely worthwhile to point out that our new approach is completely different from the previous ones, predominantly for a different partition of the Hamiltonian. Therefore, in order to better appreciate this, we will properly show in Appendix the main differences between the various ELMO based perturbative strategies.

\section{Theory}

Let us consider a $2 N_{o}$ electron closed-shell system and let us introduce a localization scheme subdividing the molecule into $s$ fragments which can overlap. Each fragment is characterized by a proper number of ELMOs that are defined as linear combination of the only basis functions centred on the atoms belonging to that subunit. Therefore, for the $i$-th fragment, the local basis-set is $\left\{\left|\chi_{i \mu}\right\rangle\right\}_{\mu=1}^{M_{i}}$ and the corresponding ELMOs are defined as:

$$
\left|\phi_{i \alpha}\right\rangle=\sum_{\mu=1}^{M_{i}} C_{i \mu, i \alpha}\left|\chi_{i \mu}\right\rangle
$$

Now, let us describe the system with a Slater determinant built up with Extremely Localized Molecular Orbitals (ELMO wavefunction):

$$
\left|\Psi_{E L M O}\right\rangle=\hat{\mathrm{A}}\left[\prod_{i=1}^{s} \prod_{\alpha=1}^{n_{i}} \phi_{i \alpha} \bar{\phi}_{i \alpha}\right]=\hat{\mathrm{A}}[\boldsymbol{\Phi}]
$$

where $\hat{\mathrm{A}}$ is the antisymmetrizer, $n_{i}$ is the number of doubly occupied ELMOs for the $i$-th fragment and $\bar{\phi}_{i \alpha}$ is a spinorbital with spatial part $\left|\phi_{i \alpha}\right\rangle$ and spin part $\beta$. It can be shown [16] that the orbitals in (1) can be obtained by solving the following equation for each fragment:

$$
\hat{f}^{(i)}\left|\phi_{i \alpha}\right\rangle=\varepsilon_{i \alpha}\left|\phi_{i \alpha}\right\rangle
$$


with $\hat{f}^{(i)}$ as the modified Fock operator for the $i$-th subunit and $\varepsilon_{i \alpha}$ as the orbital energy associated with the $\left|\phi_{i \alpha}\right\rangle$ orbital. For the sake of completeness, we show the definition of the operator $\hat{f}^{(i)}$ :

$$
\hat{f}^{(i)}=\left(1-\hat{\rho}+\hat{\rho}_{i}^{+}\right) \hat{f}\left(1-\hat{\rho}+\hat{\rho}_{i}\right)
$$

where $\hat{f}$ is the usual one-electron Fock operator, $\hat{\rho}$ is the global ELMO density operator and $\hat{\rho}_{i}$ is the ELMO density operator for the only $i$-th fragment.

So, each fragment is characterized by a set of occupied and virtual orbitals which share the same localization scheme and occupied and virtual orbitals belonging to the same fragment constitute an orthogonal set, while orbitals of different fragments are non-orthogonal. Furthermore, it is important to observe that the fragments can share atomic orbitals and this is the reason why the total number of ELMOs is greater than the total number of basis functions. Hence, using virtual ELMOs, linear dependence problems can arise and, as we will see, a proper selection of these orbitals has to be performed.

As already discussed in the introduction, due to the reduction in the number of variational parameters, the energy associated with the ELMO wavefunction is higher than the corresponding Hartree-Fock energy. So, in order to improve the description provided by $\left|\Psi_{E L M O}\right\rangle$, we propose a perturbative approach based on this Møller-Plesset type partition of the electronic Hamiltonian:

$$
\hat{H}=\hat{F}_{0}+\hat{V}=\sum_{j=1}^{2 N_{o}} \hat{f}_{0}\left(\mathbf{x}_{j}\right)+\hat{V}
$$

with $\mathbf{x}_{j}$ representing the spatial and spin coordinates of the $j$-th electron and with $\hat{f}_{0}$ defined as follows:

$$
\hat{f}_{0}=\hat{\rho} \hat{f} \hat{\rho}+(1-\hat{\rho}) \hat{f}(1-\hat{\rho})
$$


where $\hat{f}$ and $\hat{\rho}$ have been previously defined explaining equation (3).

Nevertheless, following this approach, we face two drawbacks. The first one arises because $\hat{f}$ is unknown. To overcome this problem, we can introduce the following approximation: instead of using the exact Fock operator $\hat{f}\left(\mathbf{D}_{H F}\right)$, namely the Fock operator built up with the fully converged Hartree-Fock density matrix, it is possible to construct the Fock operator by means of the fully converged ELMO density matrix $\mathbf{D}_{\text {ELMO }}$. Of course, it is obvious that:

$$
\hat{f}\left(\mathbf{D}_{\text {ELMO }}\right) \approx \hat{f}\left(\mathbf{D}_{H F}\right) \Leftrightarrow \mathbf{D}_{E L M O} \approx \mathbf{D}_{H F}
$$

The second drawback is that we do not know the eigenfunctions of the operator $\hat{f}_{0}$, but orthonormal orbitals that diagonalize $\hat{f}_{0}$ can be easily constructed following a procedure that Khaliullin et al. used for their perturbative SCF-MI method [47], which we will briefly describe below. From now on, for the sake of clarity, we will use $i$ and $j$ for occupied Molecular Orbital (MOs) indexes, $a$ and $b$ for virtual MOs indexes, $p$ and $q$ for generic MOs indexes.

As a first step, in order to avoid linear dependence problems, we select a proper set of $N_{v}$ virtual orbitals discarding the virtual ELMOs that are linear combinations of the occupied ELMOs and of the previously selected virtual orbitals. In particular, in order to accomplish this task, each virtual ELMO is Gram-Schmidt orthogonalized with respect to the occupied ELMOs and the previously selected virtual orbitals. After the orthogonalization process, we check the norm of the transformed ELMO and if it is significantly different from zero (i.e., greater than the threshold $\eta=1 \cdot 10^{-6}$ ), the original orbital, namely the ELMO as it was before the Gram-Schmidt procedure, is selected in the group of virtual orbitals to be used. Hence, after this selection we have the following set of Extremely Localized Molecular Orbitals: 


$$
\left\{\left|\phi_{p}\right\rangle\right\}_{p=1}^{N_{t}} \quad \text { with } \quad N_{t}=N_{o}+N_{v}
$$

where the corresponding coefficient matrix $\mathbf{C}_{t}$ has the first $N_{o}$ columns representing the occupied ELMOs. The selected orbitals are afterwards orthonormalized using the inverse Cholesky factor of the overlap matrix $\mathbf{S}_{t}$ between the ELMOs:

$$
\begin{aligned}
& \mathbf{S}_{t}=\mathbf{L} \mathbf{L}^{T} \\
& \mathbf{C}_{t}^{\prime}=\mathbf{C}_{t}\left(\mathbf{L}^{-1}\right)^{T}
\end{aligned}
$$

In this way, we obtain a new set of orthonormal orbitals:

$$
\left\{\left|\phi_{p}^{\prime}\right\rangle\right\}_{p=1}^{N_{t}} \quad \text { with } \quad N_{t}=N_{o}+N_{v}
$$

and it is worth to note that the occupied space is not mixed with the virtual space because $\mathbf{L}^{-1}$ has a lower triangular structure and because, as observed above, the first $N_{o}$ columns of $\mathbf{C}_{t}$ represent the occupied ELMOs.

Now, the matrix $\mathbf{f}_{0}$ associated with the operator $\hat{f}_{0}$ in the space spanned by the basis $\left\{\left|\phi_{p}^{\prime}\right\rangle\right\}_{p=1}^{N_{t}}$ is a block diagonal matrix and, diagonalizing the occupied-occupied block $\left[\mathbf{f}_{0}\right]_{o o}$ and the virtual-virtual block $\left[\mathbf{f}_{0}\right]_{v v}$ :

$$
\begin{aligned}
& {\left[\mathbf{f}_{0}\right]_{o o} \mathbf{K}^{o}=\mathbf{K}^{o} \lambda^{o}} \\
& {\left[\mathbf{f}_{0}\right]_{v v} \mathbf{K}^{v}=\mathbf{K}^{v} \lambda^{v}}
\end{aligned}
$$

we can write the new set occupied orbitals, which are eigenfunctions of $\hat{f}_{0}$, as follows:

$$
\left|\psi_{i}\right\rangle=\sum_{j=1}^{N_{o}} K_{j i}^{o}\left|\phi_{j}^{\prime}\right\rangle
$$

and the virtual ones as:

$$
\left|\psi_{a}\right\rangle=\sum_{b=1}^{N_{v}} K_{b a}^{v}\left|\phi_{b}^{\prime}\right\rangle
$$


Of course, we have:

$$
\left\langle\psi_{p}\left|\hat{f}_{0}\right| \psi_{q}\right\rangle=\lambda_{q} \delta_{p q}
$$

and, moreover, the new set $\left\{\left|\psi_{p}\right\rangle\right\}_{p=1}^{N_{t}}$ spans the same occupied and virtual subspaces as the converged non-orthogonal ELMOs.

Considering the expression of the ELMO wavefunction (1) and the eigenfunctions of the operator $\hat{f}_{0}$, we have:

$$
\begin{aligned}
\left|\Psi^{(0)}\right\rangle=\left|\Psi_{E L M O}\right\rangle & =\hat{\mathrm{A}}\left[\prod_{i=1}^{N_{o}} \psi_{i} \bar{\psi}_{i}\right]=\hat{\mathrm{A}}[\boldsymbol{\Psi}] \\
E^{(0)} & =2 \sum_{i=1}^{N_{o}} \lambda_{i}
\end{aligned}
$$

and

$$
E^{(1)}=\left\langle\Psi^{(0)}\left|\hat{H}-\hat{F}_{0}\right| \Psi^{(0)}\right\rangle=E_{E L M O}-E^{(0)}
$$

From equation (15) it is obvious that the first-order corrected energy corresponds to the ELMO energy and, therefore, to improve the ELMO description we have to consider the second-order energy correction, which can be computed by making the Hylleraas functional stationary $[51,52]$. In our case, in order to guarantee the orthogonality between $\left|\Psi^{(1)}\right\rangle$ and $\left|\Psi_{E L M O}\right\rangle$, this functional has the following form:

$$
J_{2}\left[\Psi^{(1)}\right]=2 \operatorname{Re}\left\langle\Psi^{(1)}\left|\hat{H}-\hat{F}_{0}\right| \Psi_{E L M O}\right\rangle+\left\langle\Psi^{(1)}\left|\hat{F}_{0}-E^{(0)}+\zeta \hat{P}\right| \Psi^{(1)}\right\rangle
$$

where $\zeta$ is an arbitrary real number greater than a threshold to be determined and $\hat{P}=\left|\Psi_{E L M O}\right\rangle\left\langle\Psi_{E L M O}\right|$

Furthermore, we assume that the first-order correction to the ELMO wavefunction can be expressed as a linear combination that takes into account the ELMO wavefunction itself and the structures $\left|\Psi_{i}^{a}\right\rangle$ associated with the excitations from the occupied to the virtual orbitals: 


$$
\left|\Psi^{(1)}\right\rangle=C_{0}^{(1)}\left|\Psi_{E L M O}\right\rangle+\sum_{i=1}^{N_{o}} \sum_{a=1}^{N_{v}} C_{i a}^{(1)}\left(\hat{\mathrm{A}}\left[\boldsymbol{\Psi}_{(i)} \psi_{i} \bar{\psi}_{a}\right]-\hat{\mathrm{A}}\left[\boldsymbol{\Psi}_{(i)} \bar{\psi}_{i} \psi_{a}\right]\right)
$$

with $\boldsymbol{\Psi}_{(i)}$ as the Hartree product of the doubly occupied orbitals without the couple $\psi_{i} \bar{\psi}_{i}$. In order to obtain the perturbative correction $\left|\Psi^{(1)}\right\rangle$, we have to determine the coefficients of expansion (17) which make the Hylleraas functional stationary. It is possible to show that, requiring $\delta J_{2}\left[\Psi^{(1)}\right\rfloor=0$ for any arbitrary variations $\left|\delta \Psi^{(1)}\right\rangle$ is equivalent to look for the first-order correction to the ELMO wavefunction that satisfies the following equation:

$$
\left(\hat{F}_{0}-E^{(0)}+\zeta \hat{P}\right)\left|\Psi^{(1)}\right\rangle=\left(\hat{F}_{0}-\hat{H}\right)\left|\Psi_{\text {ELMO }}\right\rangle
$$

Introducing in (18) the structures on which $\left|\Psi^{(1)}\right\rangle$ is expanded, we obtain the following matrix equation:

$$
\left(\mathbf{F}_{0}-\mathbf{E}^{(0)}+\zeta \mathbf{P}\right) \mathbf{C}^{(1)}=\left(\mathbf{F}_{0}-\mathbf{H}\right) \mathbf{C}^{(0)}
$$

and due to the structure of the matrices involved in (19), we have these simple expressions for the coefficients $C_{0}^{(1)}$ and $C_{i a}^{(1)}$ :

$$
\begin{gathered}
C_{0}^{(1)}=\frac{E^{(0)}-E_{\text {ELMO }}}{\zeta} \\
C_{i a}^{(1)}=\frac{\left\langle\Psi_{\text {ELMO }}|\hat{H}| \Psi_{i}^{a}\right\rangle}{2 E^{(0)}-\left\langle\Psi_{i}^{a}\left|\hat{F}_{0}\right| \Psi_{i}^{a}\right\rangle}
\end{gathered}
$$

where it is possible to show that:

$$
\left\langle\Psi_{\text {ELMO }}|\hat{H}| \Psi_{i}^{a}\right\rangle=2\left\langle\psi_{i}|\hat{f}| \psi_{a}\right\rangle
$$

and

$$
\left\langle\Psi_{i}^{a}\left|\hat{F}_{0}\right| \Psi_{i}^{a}\right\rangle=2\left(E^{(0)}-\lambda_{i}+\lambda_{a}\right)
$$

It is worthwhile to observe that, to have $\left|\Psi^{(1)}\right\rangle$ orthogonal to $\left|\Psi_{E L M O}\right\rangle, C_{0}^{(1)}$ must be equal to zero. Therefore, the parameter $\zeta$ must fulfil the condition $\zeta>E^{(0)}-E_{E L M O}$ (in all the 
calculations performed we set $\zeta=1 \cdot 10^{8}$ ) and, finally, the second order energy correction can be written as:

$$
E^{(2)}=2 \sum_{i=1}^{N_{o}} \sum_{a=1}^{N_{v}} C_{i a}^{(1)}\left\langle\Psi_{E L M O}|\hat{H}| \Psi_{i}^{a}\right\rangle+\sum_{i=1}^{N_{o}} \sum_{a=1}^{N_{v}}\left|C_{i a}^{(1)}\right|^{2}\left(\left\langle\Psi_{i}^{a}\left|\hat{F}_{0}\right| \Psi_{i}^{a}\right\rangle-2 E^{(0)}\right)
$$

\section{Test Calculations}

In order to test the reliability and the capabilities of our new strategy, we have computed some rotational profiles for the ethanolamine and dimethylacetamide molecules, which are shown in Figure 1A and Figure 1B, respectively. To determine the rotational profiles at different levels of theory, we first optimized the molecular geometries at the MP2/6-311G** level constraining the selected dihedral angle to a specific value from $0^{\circ}$ to $360^{\circ}$ with a $15^{\circ}$ step. Afterwards, for each optimized molecular geometry, we performed single point calculations using the $6-311 \mathrm{G}^{* *}$ basis-set at the Restricted Hartree-Fock (RHF), ELMO and Perturbative ELMO (ELMO-Pert) levels. In particular, for all the ELMO-Pert calculations we have used the maximum allowed number of excitations, namely 1717 for ethanolamine and 3456 for dimethylacetamide. Finally, for the sake of completeness, it is worth pointing out that each ELMO calculation has been carried out using the most localized partitioning scheme corresponding to the Lewis molecular structure and that, except for the Peturbative ELMO method, all the computations have been performed using the GAMESS-UK package [21].

Now, let us consider individually the three ethanolamine rotational profiles corresponding to the three dihedral angles we examined: N9-C1-C4-O7, H10-N9-C1-C4, H8-O7-C4-C1. In the first case, see Figure 2, the ELMO method is not able to reproduce the RHF profile, while the perturbative technique yields a significant improvement. This can be further seen by analyzing Table I where, for almost all the dihedral angle values, the ELMO-Pert energy differences are closer to the RHF ones than those obtained from ELMO computations only. 
Next, considering the second dihedral angle (see Figure 3 and Table I), it is evident that the ELMO wavefunction reproduces the Hartree-Fock results only qualitatively, while the perturbative approach significantly improves the description. The third case is much more interesting (see Figure 4 and Table I). In fact, in this case the ELMO rotational profile is not even able to qualitatively reproduce the RHF picture, but, even with this bad starting point, using the ELMO-Pert method we obtain a very good agreement with the reference HartreeFock values.

In order to further investigate the performance of the new strategy, we have also considered the rotational profile associated with rotation around the amide bond in dimethylacetamide (see dihedral angle C8-N7-C5-O6 in Figure 1B). In Figure 5 and Table II it is easy to observe that the ELMO method fails. The reason for this failure is probably the inadequate localization scheme we adopted that, as mentioned above, corresponds to the molecular Lewis structure and consequently is not able to take into account the partial double bond character of the amide bond. Nevertheless, also in this situation, although the ELMO wavefunction is a very poor starting point, the new perturbative strategy improves the description and much better results are obtained.

\section{Conclusions and Future Outlooks}

From the preliminary tests performed on small systems, the new Perturbative ELMO strategy appears reliable in approaching Hartree-Fock results and we are confident that this will be confirmed by carrying out calculations on larger molecules. Furthermore, we believe that the new technique is potentially a powerful tool to perform large system calculations reaching Hartree-Fock accuracy but with a lower computational cost. Of course, this is true provided that it is easy to obtain the starting ELMO wavefunction, which can be done if we have a database of Extremely Localized Molecular Orbitals previously determined on model 
molecules and that can be readily transferred to the target system. DENPOL $[26,28]$ is a step forward in this direction. In this way we can skip SCF cycles and we need only to relax the ELMO wavefunction.

Through an analysis of the equations in the theory section, it is possible to observe that, as the system size increases, the steps that become more and more computationally expensive are the evaluation of the virtual-virtual block $\left[\mathbf{f}_{0}\right]_{v v}$, its diagonalization and the calculation of the matrix elements (22). These steps scale as $N_{v}{ }^{3}, N_{v}{ }^{3}$ and $N_{o} N_{v} N_{s}$, respectively, where $N_{s}$ is the number of single excitations taken into account in expansion (17). Hence, it is clear that, in order to decrease the computational cost, strategies that help to reduce the number of virtual orbitals and the number of single excitations should be considered. A possible development in these directions consists in the optimization of the virtual orbital space and in the definition of a topological criterion for the selection of the most important excitations, similar to what has already been introduced in the ELMO-VB method [24, 25, 28].

Finally, analyzing equation (4), we can observe that the adopted partition of the Hamiltonian allows to introduce correlation effects, but in this paper, due to the fact that we chose to expand the electronic wavefunction only considering single excitations (see equation (17)), we were able to recover only delocalization and relaxation terms. Nevertheless, this opens the possibility to develop a new perturbative method based on Localized Molecular Orbitals. Of course, the introduction of double excitations in expansion (17) will be essential to improve the performances of the present strategy and to go beyond the delocalization and relaxation energy, but, although the computational cost will increase, we believe that the extremely localized nature of the involved orbitals will allow for the introduction of simplifications into the calculations. 


\section{Acknowledgments}

We thank the NSF (MCB-0211639) for financial support. We would like to thank Prof. Hendrik J. Monkhorst (QTP, University of Florida), Dr. Andrew Taube (QTP, University of Florida) and Prof. Roberto Cammi (Università di Parma, Italy) for helpful discussions.

\section{Appendix}

As already mentioned in the introduction, our new method, although similar to previous ELMO based perturbative techniques [33-40, 45-48], it is completely different from them, predominantly because of a different partition of the Hamiltonian. Therefore, the goal of the appendix is to briefly outline the differences between these various similar strategies, and, in particular, the comparison between our technique and the PCILO methods will be stressed.

The PCILO strategies should be classified into the Original PCILO (o-PCILO) [33-38] and the Modified PCILO (m-PCILO) [39, 40]. The common idea to these two techniques is to perform a Perturbative Configuration Interaction starting from a single determinant wavefunction constructed with Extremely Localized Molecular Orbitals. It is worth noting that the ELMOs used in the PCILO approaches are different from ours. The PCILO-type ELMOs are obtained by solving Roothaan equations modified through the introduction of semiempirical approximations. On the other hand, our ELMOs satisfy the Stoll equations [16], which are fully $a b$ initio relations. It is important to note that both the PCILO-type ELMOs and the Stoll-type ELMOs are not eigenfunctions of the exact one-electron Fock operator.

For the sake of clarity, we have to point out that, in the following of this appendix, we will consider three types of molecular orbitals / spinorbitals: the PCILO-type ELMOs and the corresponding spinorbitals [33-40], which we will write as $\left|\phi_{q}^{[P]}\right\rangle$ and $\left|q^{[P]}\right\rangle$, respectively; the Nagata-type ELMOs and spinorbitals $[45,46]$, which we will indicate as $\left|\phi_{q}^{[N]}\right\rangle$ and 
$\left|q^{[N]}\right\rangle$, respectively; the working orbitals and spinorbitals in our technique, namely the eigenfunctions of operator $\hat{f}_{0}$ defined in equation (5), which we will simply write as $\left|\psi_{q}\right\rangle$ and $\left|q^{[K]}\right\rangle$, respectively. Furthermore, still considering a $2 N_{o}$ electron closed-shell system, the matrix elements of the one-electron Fock operator $\hat{f}$ in the basis of the spinorbitals $\left\{\left|q^{[X]}\right\rangle\right\}$ (with $X=P, N$ or $K$ ) are defined as follows:

$$
f_{p q}^{[X]}=\left\langle p^{[X]}|\hat{f}| q^{[X]}\right\rangle=\left\langle p^{[X]}|\hat{h}| q^{[X]}\right\rangle+\sum_{k=1}^{2 N_{o}}\left\langle p^{[X]} k^{[X]} \| q^{[X]} k^{[X]}\right\rangle
$$

Now, let us examine the different partitions of the Hamiltonian. In the o-PCILO strategy the Epstein-Nesbet partitioning [53, 54] is adopted and, therefore, this technique is completely different from our strategy.

The m-PCILO method distinguishes from the older o-PCILO because this partition is proposed:

$$
\hat{H}=\hat{H}_{0}^{[P]}+\hat{V}^{[P]}
$$

where, using the second quantization formalism and taking into account that this strategy exploits the ZDO approximation (namely, this strategy neglects the non-orthogonality of the ELMOs), we have:

$$
\begin{aligned}
\hat{H}_{0}^{[P]} & =\sum_{i=1}^{2 N_{o}}\left(\hat{i}^{[P]}\right)^{+} f_{i i}^{[P]} \hat{i}^{[P]}+\sum_{a=1}^{N_{v}}\left(\hat{a}^{[P]}\right)^{+} f_{a a}^{[P]} \hat{a}^{[P]} \\
\hat{V}^{[P]} & =\sum_{i, j=1}^{2 N_{o}}\left(\hat{i}^{[P]}\right)^{+} f_{i j}^{[P]} \hat{j}^{[P]}+\sum_{a, b=1}^{N_{v}} \cdot\left(\hat{a}^{[P]}\right)^{+} f_{a b}^{[P]} \hat{b}^{[P]}+ \\
& +\sum_{i=1}^{2 N_{o}} \sum_{a=1}^{N_{v}}\left\{\left(\hat{i}^{[P]}\right)^{+} f_{i a}^{[P]} \hat{a}^{[P]}+\left(\hat{a}^{[P]}\right)^{+} f_{a i}^{[P]} \hat{i}^{[P]}\right\}+\hat{W}
\end{aligned}
$$

with $\hat{W}$ as the two-electron contribution to the perturbation and the prime in the first two summations of $\hat{V}^{[P]}$ indicating that the terms $i=j$ and $a=b$ should not be counted, 
respectively. In other words, in the unperturbed Hamiltonian $\hat{H}_{0}^{[P]}$ only the diagonal elements of the one-electron Fock operator are considered, while the off-diagonal terms are included in the perturbation. It is extremely important to observe that this partitioning is not invariant to rotations within the occupied space and to rotations within the virtual space.

Due to this partition, and considering only single excitations as in our perturbative approach, for the m-PCILO one obtains:

$$
\begin{gathered}
E^{(0)}+E^{(1)}=E_{\text {ELMO }}^{\text {PCILO }} \\
E^{(2)}=2 \sum_{i=1}^{N_{o}} \sum_{a=1}^{N_{v}} \frac{\left|\left\langle\phi_{i}{ }^{[P]}|\hat{f}| \phi_{a}^{[P]}\right\rangle\right|^{2}}{\left\langle\phi_{i}{ }^{[P]}|\hat{f}| \phi_{i}{ }^{[P]}\right\rangle-\left\langle\phi_{a}^{[P]}|\hat{f}| \phi_{a}^{[P]}\right\rangle}
\end{gathered}
$$

Let us examine our Perturbative ELMO technique. Using the second quantization formalism, the unperturbed operator and the perturbation (see equation (4)) can be expressed like this:

$$
\begin{gathered}
\hat{F}_{0}=\sum_{i=1}^{2 N_{o}}\left(\hat{i}^{[K]}\right)^{+} \lambda_{i} \hat{i}^{[K]}+\sum_{a=1}^{N_{v}}\left(\hat{a}^{[K]}\right)^{+} \lambda_{a} \hat{a}^{[K]} \\
\hat{V}=\sum_{i=1}^{2 N_{o}}\left(\hat{i}^{[K]}\right)^{+}\left(f_{i i}^{[K]}-\lambda_{i}\right) \hat{i}^{[K]}+\sum_{a=1}^{N_{v}}\left(\hat{a}^{[K]}\right)^{+}\left(f_{a a}^{[K]}-\lambda_{a}\right) \hat{a}^{[K]}+\sum_{i, j=1}^{2 N_{o}}\left(\hat{i}^{[K]}\right)^{+} f_{i j}^{[K]} \hat{j}^{[K]}+ \\
+\sum_{a, b=1}^{N_{v}}\left(\hat{a}^{[K]}\right)^{+} f_{a b}^{[K]} \hat{b}^{[K]}+\sum_{i=1}^{2 N_{o}} \sum_{a=1}^{N_{v}}\left\{\left(\hat{i}^{[K]}\right)^{+} f_{i a}^{[K]} \hat{a}^{[K]}+\left(\hat{a}^{[K]}\right)^{+} f_{a i}^{[K]} \hat{i}^{[K]}\right\}+\hat{W}
\end{gathered}
$$

where $\lambda_{q}$ is the generic $q$-th eigenvalue of the operator $\hat{f}_{0}$ and where $\hat{W}$ and the prime in the third and in the fourth summation of $\hat{V}$ have the same meaning as in equation (28). This partition, unlike the one adopted in the m-PCILO approach, is invariant to rotations within the occupied space and to rotations within the virtual space and it leads to equations (14), (15) and to equation (24) which can be rewritten like this:

$$
E^{(2)}=2 \sum_{i=1}^{N_{o}} \sum_{a=1}^{N_{v}} \frac{\left|\left\langle\psi_{i}|\hat{f}| \psi_{a}\right\rangle\right|^{2}}{\left\langle\psi_{i}\left|\hat{f}_{0}\right| \psi_{i}\right\rangle-\left\langle\psi_{a}\left|\hat{f}_{0}\right| \psi_{a}\right\rangle}
$$


It is worthwhile to observe that the arguments of the summations in (30) and (33) are not equal. In fact, different types of orbitals are involved, the matrix elements of the one-electron Fock operator in (30) are evaluated introducing semiempirical approximations and, above all, in the denominator of equation (33) we have the operator $\hat{f}_{0}$ instead of $\hat{f}$. Hence, it is obvious that our strategy also differs from the m-PCILO method.

Now, we will briefly consider the recent perturbative techniques developed in the framework of Stoll-type ELMO equations [16], among which we also include the relations proposed by Gianinetti [49, 50] and Nagata [45, 46] for their SCF-MI and LP SCF-MI methods, respectively.

The first attempt to improve the electronic structure description provided by an ELMO wavefunction constructed with orbitals that are solutions of Stoll-type equations is the one proposed by Iwata and Nagata within the LP SCF-MI strategy $[45,46]$. In this case, although the ELMOs are not canonical, they use the following Møller-Plesset type of partitioning:

$$
\hat{H}=\hat{H}_{0}^{[N]}+\hat{V}^{[N]}
$$

with

$$
\hat{H}_{0}^{[N]}=\sum_{i, j=1}^{2 N_{o}}\left(\hat{i}^{[N]}\right)^{+} f_{i j}^{[N]} \hat{j}^{[N]}+\sum_{a, b=1}^{N_{v}}\left(\hat{a}^{[N]}\right)^{+} f_{a b}^{[N]} \hat{b}^{[N]}
$$

This choice distinguishes the technique from those shown above and, moreover, it is necessary to observe that, unlike the m-PCILO strategy, the non-orthogonality of the ELMOs is taken into account and it leads to more complicated equations. A similar perturbation method with non-canonical orbitals is the local MP2 strategy [55], but in the present case, unlike the original local MP2, the occupied orbitals are not either canonical or orthogonal. In this context, the second approach developed is the one by Khaliullin et al. [47, 48]. This method adopts the same unperturbed operator that we consider in our new perturbation 
strategy, but it is extremely important to observe that they do not partition the electronic Hamiltonian, but the Fock operator, namely they set up the perturbative approach as follows:

$$
\hat{F}=\hat{F}_{0}+\hat{V}^{[K]}=\sum_{j=1}^{2 N_{o}} \hat{f}_{0}\left(\mathbf{x}_{j}\right)+\sum_{j=1}^{2 N_{o}} \hat{v}\left(\mathbf{x}_{j}\right)
$$

where $\hat{f}_{0}$ is defined as in equation (5) and

$$
\hat{v}=\hat{\rho} \hat{f}(1-\hat{\rho})+(1-\hat{\rho}) \hat{f} \hat{\rho}
$$

with, also in this case, $\hat{\rho}$ and $\hat{f}$ as the global ELMO density operator and the one-electron Fock operator, respectively. Therefore, the Khaliullin perturbation series converges to the exact eigenvalue of the Fock operator and, hence, it is also evident that this strategy differs from the ones previously described.

Finally, we have to mention that Surján and Mayer have also developed a perturbation technique to relax the ELMO wavefunction [42-44]. This strategy is based on a completely different philosophy with respect to the approaches previously analyzed in the appendix and, in particular, it consists in computing, within the one-electron approximation, the "orthogonalization tails" for each occupied Extremely Localized Molecular Orbital as perturbative corrections. Therefore, a new set of Molecular Orbitals is obtained and this new set is used to construct a new Slater determinant whose energy is a strict upper bound to the Hartree-Fock energy. 


\section{References}

1. S. Goedecker, Rev. Mod. Phys. 71, 1085 (1999).

2. W. Yang, Phys. Rev. Lett. 66, 1438 (1991).

3. W. Yang, T.-S. Lee, J. Chem. Phys. 103, 5674 (1995).

4. S. L. Dixon, K. M. Merz Jr., J. Chem. Phys. 104, 6643 (1996).

5. S. L. Dixon, K. M. Merz Jr., J. Chem. Phys. 107, 879 (1997).

6. S. R. Gadre, R. N. Shirsat, A. C. Limaye, J. Phys. Chem. 98, 9165 (1994).

7. K. Babu, S. R. Gadre, J. Comput. Chem. 24, 484 (2003).

8. K. Babu, V. Ganesh, S. R. Gadre, N. E. Ghermani, Theor. Chem. Acc. 111, 255 (2004).

9. T. E. Exner, P. G. Mezey, J. Phys. Chem. A 106, 11791 (2002).

10. T. E. Exner, P. G. Mezey, J. Comput. Chem. 24, 1980 (2003).

11. Z. Szekeres, P. G. Mezey, P. R. Surján, Chem. Phys. Lett. 424, 420 (2006).

12. W. Kohn, Phys. Rev. Lett. 76, 3168 (1996).

13. E. Prodan, W. Kohn, Proc. Natl. Acad. Sci. USA 102, 11635 (2005).

14. E. Prodan, Phys. Rev. B 73, 085108 (2006).

15. J. Pipek, P. G. Mezey, J. Chem. Phys. 90, 4916 (1989).

16. H. Stoll, G. Wagenblast, H. Preuss, Theoret. Chim. Acta 57, 169 (1980).

17. Z. Szekeres, P. R. Surján, Chem. Phys. Lett. 369, 125 (2003).

18. M. Sironi, A. Famulari, Theor. Chem. Acc. 103, 417 (2000).

19. G. F. Smits, C. Altona, Theoret. Chim. Acta 67, 461 (1985).

20. A. Fornili, M. Sironi, M. Raimondi, J. Mol. Struct. (Theochem) 632, 157 (2003).

21. M. F. Guest, I. J. Bush, H. J. J. van Dam, P. Sherwood, J. M. H. Thomas, J. H. van Lenthe, R. W. A. Havenith, J. Kendrick, Mol. Phys. 103, 719 (2005).

22. M. Sironi, A. Famulari, M. Raimondi, S. Chiesa, J. Mol. Struct. (Theochem) 529, 47 (2000). 
23. E. Burresi, M. Sironi, Theor. Chem. Acc. 112, 247 (2004).

24. A. Genoni, M. Sironi, Theor. Chem. Acc. 112, 254 (2004).

25. A. Genoni, A. Fornili, M. Sironi, J. Comput. Chem. 26, 827 (2005).

26. M. Ghitti, A. Genoni, M. Civera, A. Fornili, S. Pieraccini, M. Sironi, in: Proceedings of the International Karlsruhe Nanoscience Workshop, University of Karlsruhe, 2005.

27. A. Genoni, M. Ghitti, S. Pieraccini, M. Sironi, Chem. Phys. Lett. 415, 256 (2005).

28. M. Sironi, A. Genoni, M. Civera, S. Pieraccini, M. Ghitti, Theor. Chem. Acc. 117, 685 (2007).

29. A. Fornili, Y. Moreau, M. Sironi, X. Assfeld, J. Comput. Chem. 27, 515 (2006).

30. A. Fornili, P. F. Loos, M. Sironi, X. Assfeld, Chem. Phys. Lett. 427, 236 (2006).

31. X. Assfeld, J. L. Rivail, Chem. Phys. Lett. 263, 100 (1996).

32. N. Ferrè, X. Assfeld, J. L. Rivail, J .Comput. Chem. 23, 610 (2002).

33. S. Diner, J. P. Malrieu, P. Claverie, F. Jordan, Chem. Phys. Lett. 2, 319 (1968).

34. S. Diner, J. P. Malrieu, P. Claverie, Theoret. Chim. Acta 13, 1 (1969).

35. J. P. Malrieu, P. Claverie, S. Diner, Theoret. Chim. Acta 13, 18 (1969).

36. S. Diner, J. P. Malrieu, F. Jordan, M. Gilbert, Theoret. Chim. Acta 15, 100 (1969).

37. F. Jordan, M. Gilbert, J. P. Malrieu, U. Pincelli, Theoret. Chim. Acta 15, 211 (1969).

38. R. Bonaccorsi, C. Petrongolo, E. Scrocco, J. Tomasi, Theoret. Chim. Acta 15, 332 (1969).

39. R. Boča, P. Pelikán, Theoret. Chim. Acta 50, 11 (1978).

40. R. Boča, Theoret. Chim. Acta 61, 179 (1982).

41. P. R. Surján, M. Révész, I. Mayer, J. Chem. Soc., Faraday Trans. II 77, 1129 (1981).

42. P. R. Surján, I. Mayer, Theoret. Chim. Acta 59, 603 (1981).

43. P. R. Surján, I. Mayer, M. Kertész, J. Chem. Phys. 77, 2454 (1982).

44. I. Mayer, P. R. Surján, J. Chem. Phys. 80, 5649 (1984).

45. T. Nagata, S. Iwata, J. Chem. Phys. 120, 3555 (2004). 
46. S. Iwata, T. Nagata, Theor. Chem. Acc. 117, 137 (2007)

47. R. Z. Khaliullin, M. Head-Gordon, A. T. Bell, J. Chem. Phys. 124, 204105 (2006).

48. R. Z. Khaliullin, E.A. Cobar, R. C. Lochan, A. T. Bell, M. Head-Gordon, J.Phys. Chem. A 111, 8753 (2007).

49. E. Gianinetti, M. Raimondi, E. Tornaghi, Int. J. Quantum Chem. 60, 157 (1996).

50. E. Gianinetti, I. Vandoni, A. Famulari, M. Raimondi, Adv. Quantum Chem. 31, 251 (1998).

51. E. A. Hylleraas, Z. Phys 65, 209 (1930).

52. H. E. Bethe, E. E. Salpeter, Quantum Mechanichs of One- and Two-Electron Atoms, Springer, Berlin, 1957.

53. P. S. Epstein, Phys. Rev. 28, 695 (1926)

54. R. K. Nesbet, Proc. Roy. Soc. (London) A230, 312 (1955).

55. P. Pulay, S. Saebo, Theoret. Chim. Acta 69, 357 (1986). 


\section{Figure Captions}

Figure 1. (Color online). Ethanolamine (A) and Dimethylacetamide (B) molecules.

Figure 2. (Color online). Ethanolamine rotational profiles associated with the N9-C1-C4-O7 dihedral angle. For each profile the energy differences are referred to the minimum energy conformation obtained with the method represented by the curve itself.

Figure 3. (Color online). Ethanolamine rotational profiles associated with the H10-N9-C1-C4 dihedral angle. For each profile the energy differences are referred to the minimum energy conformation obtained with the method represented by the curve itself.

Figure 4. (Color online). Ethanolamine rotational profiles associated with the H8-O7-C4-C1 dihedral angle. For each profile the energy differences are referred to the minimum energy conformation obtained with the method represented by the curve itself.

Figure 5. (Color online). Dimethylacetamide rotational profiles associated with the C8-N7-C5-O6 dihedral angle. For each profile the energy differences are referred to the minimum energy conformation obtained with the method represented by the curve itself. 
Table I - Ethanolamine rotational profiles (N9-C1-C4-O7, H10-N9-C1-C4 and H8-O7-C4-C1 dihedral angles): deviations of the ELMO and ELMO-Pert energy differences from the corresponding RHF $\Delta$ Es. For each dihedral angle the energy differences are referred to the minimum energy conformation at the MP2/6-311G** level $\left(60^{\circ}\right.$ for N9-C1-C4-O7, $195^{\circ}$ for $\mathrm{H} 10-\mathrm{N} 9-\mathrm{C} 1-\mathrm{C} 4$ and $315^{\circ}$ for H8-O7-C4-C1) ${ }^{(a, b)}$.

\begin{tabular}{|c|c|c|c|c|c|c|}
\hline \multirow{2}{*}{ Dihedral Angle $\left(^{\circ}\right)$} & \multicolumn{2}{|c|}{ N9-C1-C4-O7 } & \multicolumn{2}{|c|}{ H10-N9-C1-C4 } & \multicolumn{2}{|c|}{$\mathrm{H} 8-\mathrm{O} 7-\mathrm{C} 4-\mathrm{C} 1$} \\
\hline & ELMO & ELMO-Pert & ELMO & ELMO-Pert & ELMO & ELMO-Pert \\
\hline 0 & -0.79 & -0.11 & -0.40 & -0.20 & 0.06 & 0.09 \\
\hline 15 & 0.09 & 0.14 & -0.23 & -0.16 & -0.04 & 0.02 \\
\hline 30 & 0.54 & 0.24 & -0.69 & -0.23 & 0.30 & 0.02 \\
\hline 45 & 0.50 & 0.19 & -0.21 & -0.08 & 0.80 & 0.07 \\
\hline 60 & 0.00 & 0.00 & 0.02 & -0.01 & 1.07 & 0.09 \\
\hline 75 & 0.40 & -0.11 & 0.03 & 0.01 & 1.07 & 0.05 \\
\hline 90 & -0.67 & -0.37 & -0.23 & -0.05 & 0.83 & -0.04 \\
\hline 105 & -1.39 & -0.52 & -0.71 & -0.17 & 0.52 & -0.13 \\
\hline 120 & -1.51 & -0.53 & -1.22 & -0.32 & 0.38 & -0.18 \\
\hline 135 & -1.15 & -0.43 & -1.51 & -0.41 & 0.36 & -0.20 \\
\hline 150 & -0.65 & -0.31 & -1.49 & -0.43 & 0.53 & -0.16 \\
\hline 165 & -0.26 & -0.19 & -1.30 & -0.40 & 0.67 & -0.12 \\
\hline 180 & -0.24 & -0.16 & -1.10 & -0.34 & 0.64 & -0.10 \\
\hline 195 & 0.15 & -0.07 & 0.00 & 0.00 & 0.41 & -0.14 \\
\hline 210 & -1.23 & -0.38 & -0.13 & -0.01 & 0.05 & -0.21 \\
\hline 225 & -1.84 & -0.51 & -0.62 & -0.13 & -0.55 & -0.32 \\
\hline 240 & -2.25 & -0.62 & -1.17 & -0.29 & -0.70 & -0.34 \\
\hline 255 & -1.74 & -0.53 & -1.50 & -0.41 & -0.91 & -0.37 \\
\hline 270 & -1.31 & -0.43 & -1.47 & -0.43 & -0.93 & -0.35 \\
\hline 285 & -1.10 & -0.35 & -1.22 & -0.37 & -0.76 & -0.28 \\
\hline 300 & -1.23 & -0.37 & -0.93 & -0.35 & -0.45 & -0.16 \\
\hline 315 & 0.48 & 0.18 & -0.89 & -0.30 & 0.00 & 0.00 \\
\hline 330 & 0.57 & 0.25 & -0.63 & -0.25 & 0.27 & 0.11 \\
\hline 345 & -1.18 & -0.25 & -0.53 & -0.24 & 0.27 & 0.14 \\
\hline
\end{tabular}

(a) Acronyms are defined in the text.

(b) All the energy values are expressed in $\mathrm{kcal} / \mathrm{mol}$. 
Table II - Dimethylacetamide rotational profile (C8-N7-C5-O6 dihedral angle): deviations of the ELMO and ELMO-Pert energy differences from the corresponding RHF $\triangle$ Es. The energy differences are referred to the minimum energy conformation at the MP2/6-311G** level (dihedral angle equal to $\left.165^{\circ}\right)^{(a, b)}$

\begin{tabular}{|c|c|c|}
\hline \multirow{2}{*}{ Dihedral Angle $\left(^{\circ}\right)$} & \multicolumn{2}{|c|}{ C8-N7-C5-O6 } \\
\hline & ELMO & ELMO-Pert \\
\hline 0 & 2.06 & 0.82 \\
\hline 15 & -1.13 & -0.44 \\
\hline 30 & -3.69 & -1.43 \\
\hline 45 & -16.55 & -6.89 \\
\hline 60 & -17.73 & -7.37 \\
\hline 75 & -17.14 & -7.10 \\
\hline 90 & -15.35 & -6.33 \\
\hline 105 & -12.55 & -5.04 \\
\hline 120 & -9.77 & -3.91 \\
\hline 135 & -6.47 & -2.59 \\
\hline 150 & -3.21 & -1.29 \\
\hline 180 & 2.60 & 1.02 \\
\hline 195 & 0.00 & -0.01 \\
\hline 210 & -3.11 & -1.25 \\
\hline 225 & -6.46 & -2.58 \\
\hline 240 & -9.67 & -3.86 \\
\hline 255 & -12.72 & -5.11 \\
\hline 270 & -15.36 & -6.31 \\
\hline 285 & -17.11 & -7.13 \\
\hline 300 & $-10,02$ & -3.94 \\
\hline 315 & $-6,70$ & -2.60 \\
\hline 330 & $-3,91$ & -1.52 \\
\hline 345 & $-1,11$ & -0.43 \\
\hline
\end{tabular}

(a) Acronyms are defined in the text.

(b) All the energy values are expressed in $\mathrm{kcal} / \mathrm{mol}$. 
Figure 1-

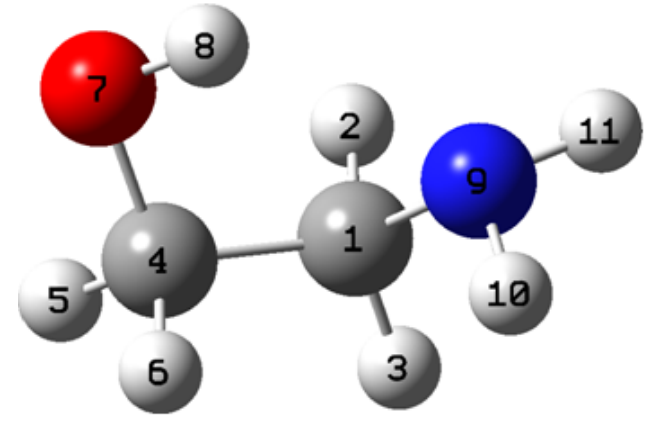

A

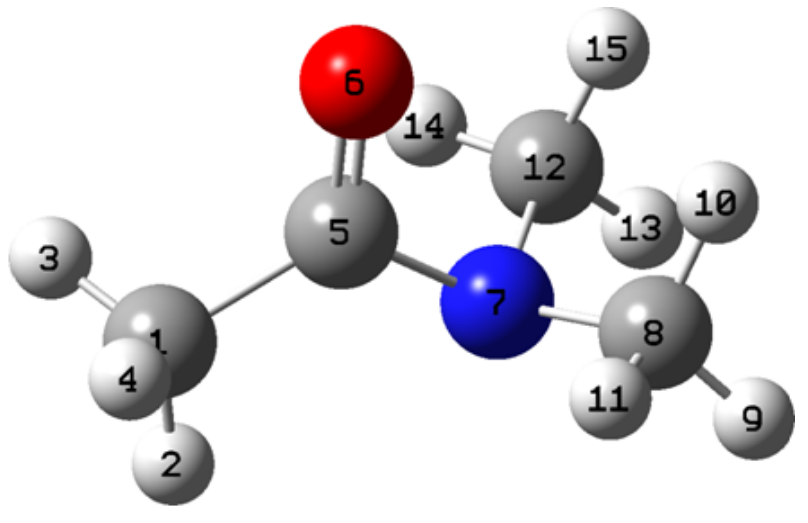

B 
Figure 2 -

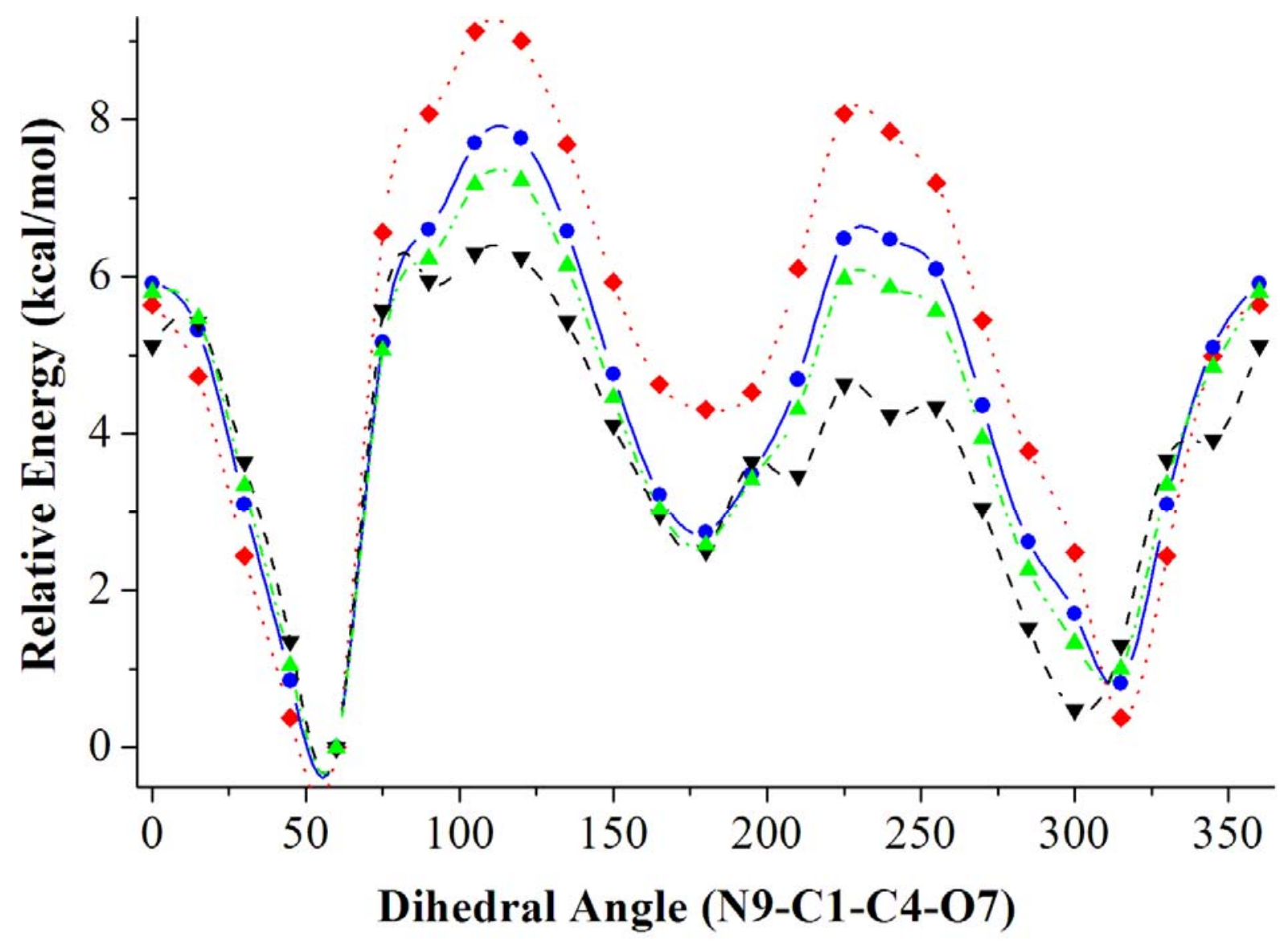

$\bullet$ MP2 $\quad-\bullet-$ RHF $\quad-$ - ELMO $\quad-\Delta-$ ELMO-Pert 
Figure 3-

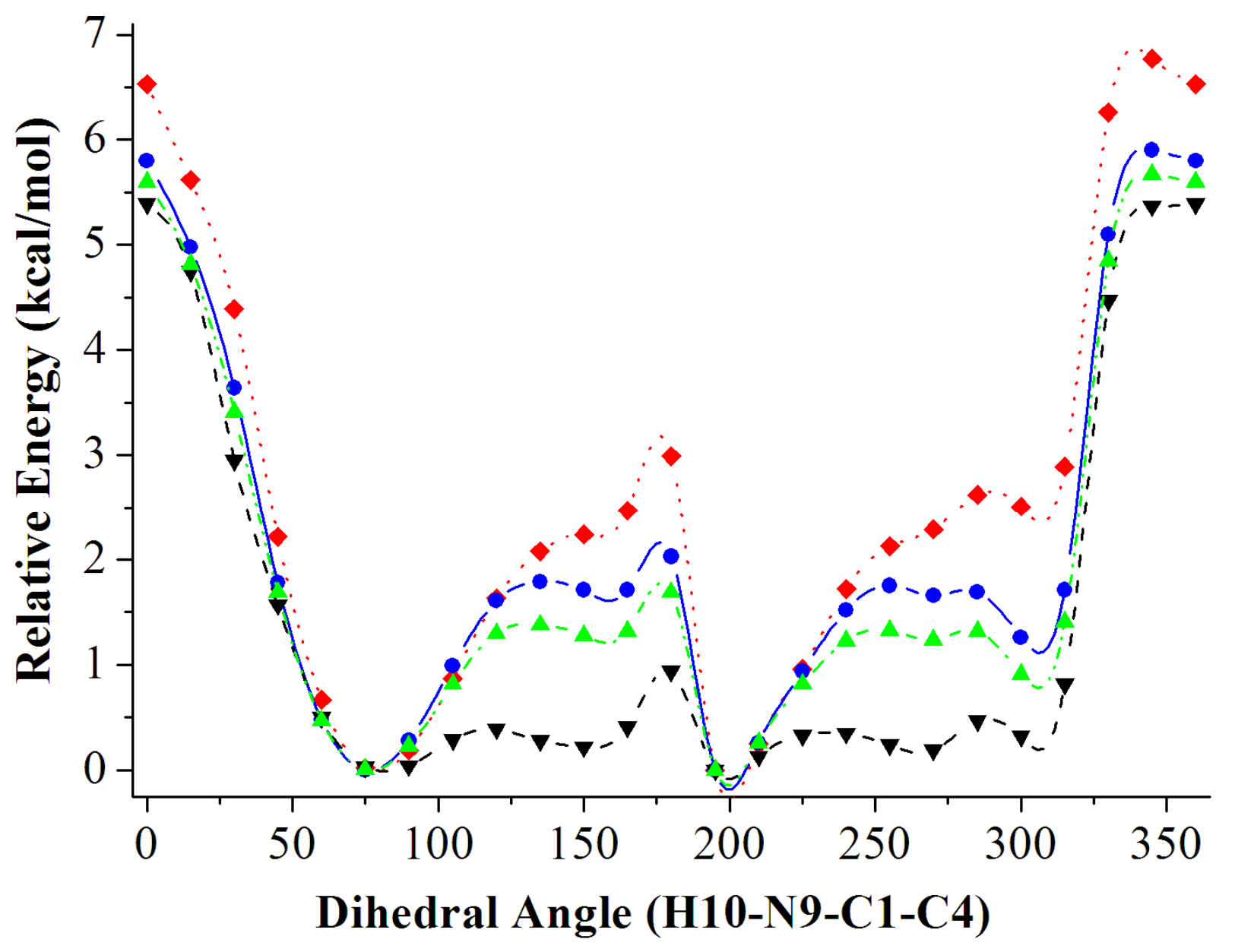

$\cdots$ MP2 $-\bullet-$ RHF $-\nabla-$ ELMO $\quad$-.-. ELMO-Pert 
Figure 4-

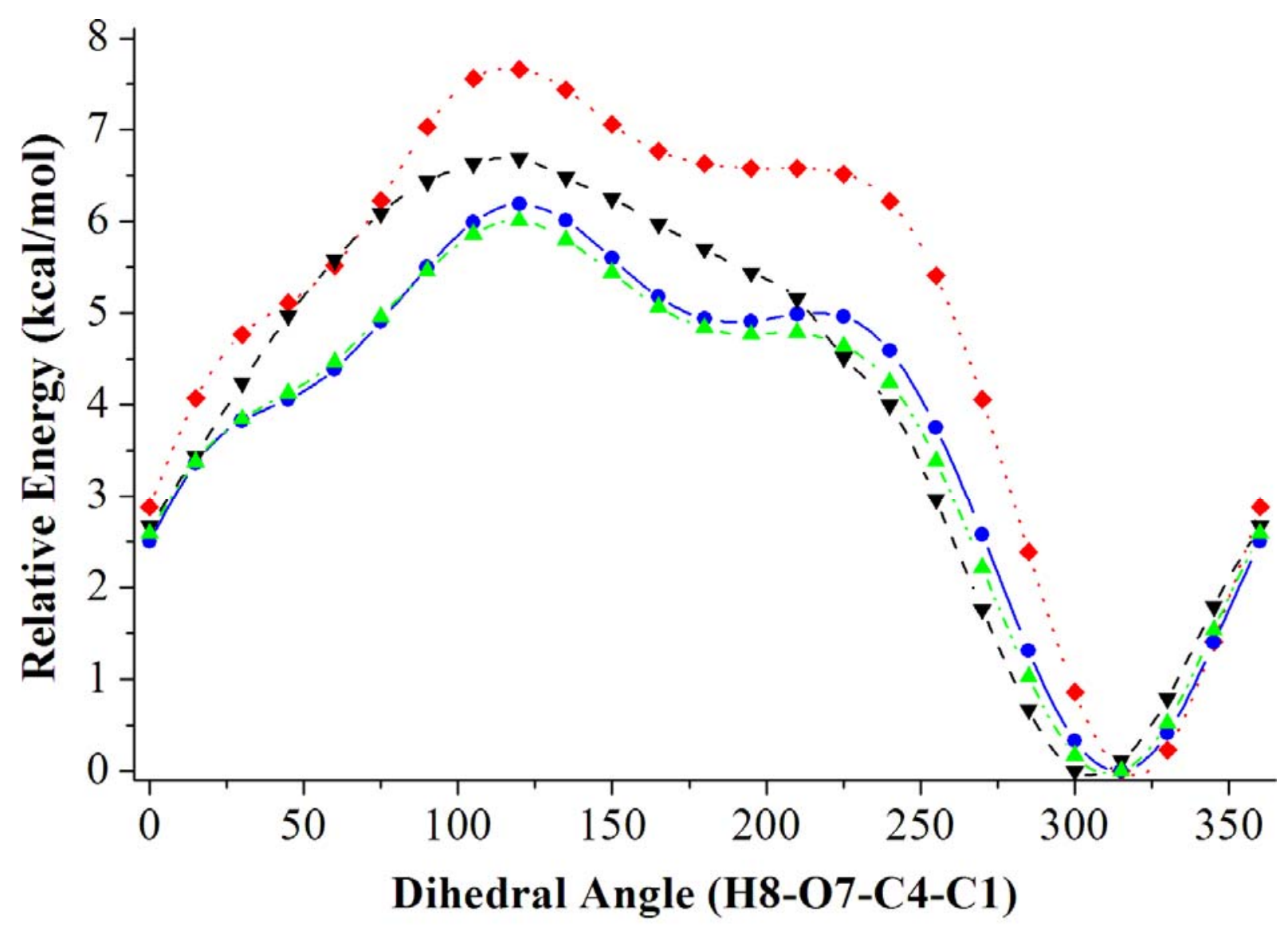

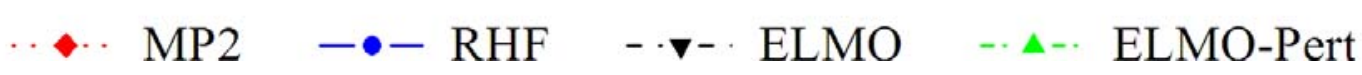


Figure 5-

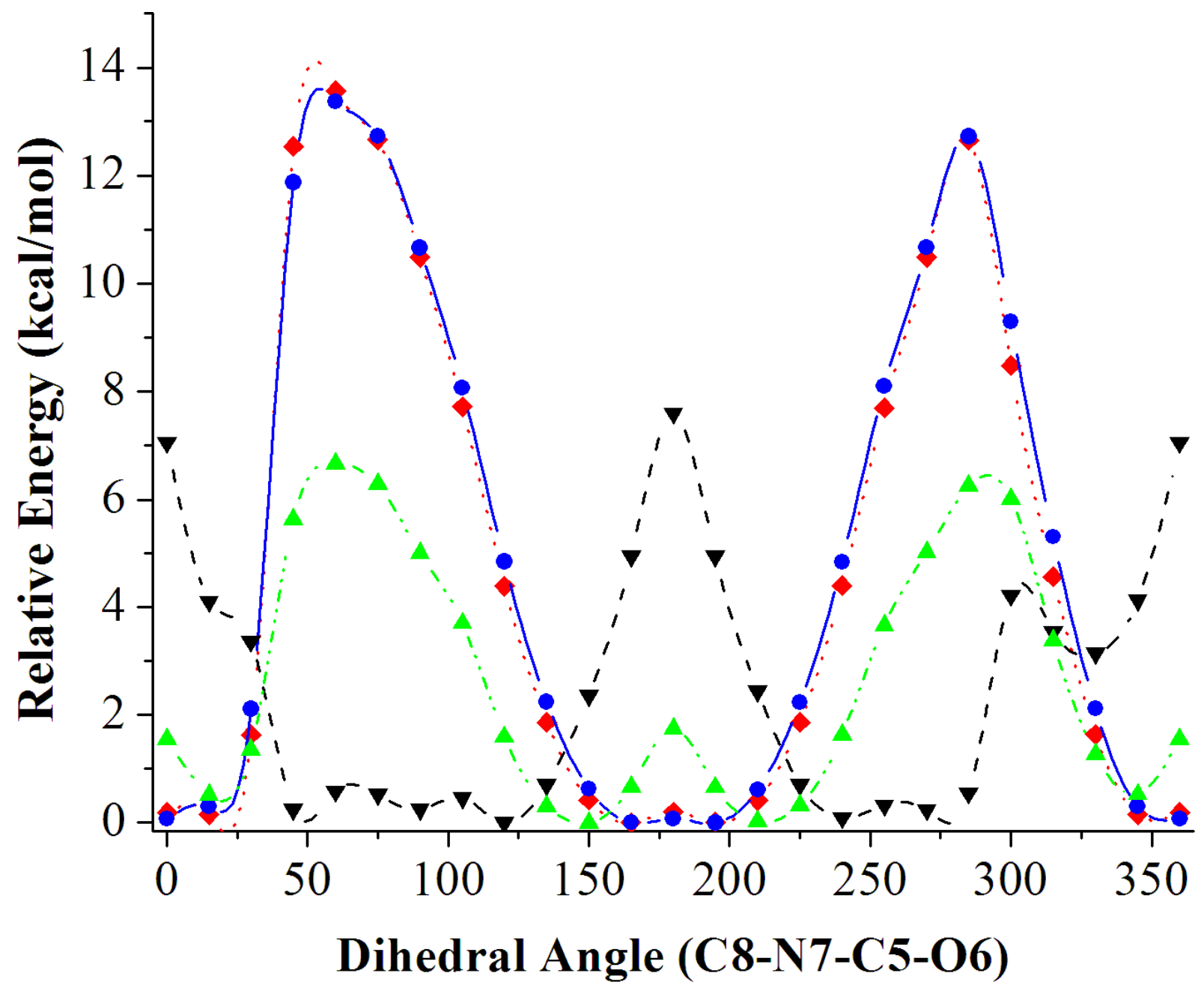

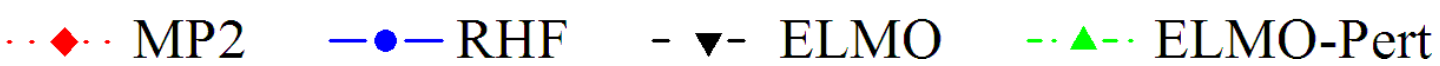

